\title{
SEISMIC BEHAVIOUR OF VARYING FLOORS OF RC CONVENTIONAL AND MONOLITHIC BUILDING
}

\author{
Chinmayi M \\ P.G. student \\ Department of civil Engineering \\ Malnad college of Engineering, Hassan, \\ Karnataka, India.
}

\author{
Dr.H S Narashimhan \\ Associate professor \\ Department of Civil Engineering \\ Malnad College of Engineering, Hassan \\ Karnataka, India
}

\author{
G Ambrish \\ Executive Director \\ Nixit Consultants Pvt.Ltd. \\ Bengaluru, Karnataka, India
}

\begin{abstract}
RC structures are traditionally modelled by assuming that all the loads are applied in one step. This assumption does not reflect the structure's actual behaviour. Here we study the variations in load and seismic response of structure at each stage of construction. A 24 storeys Conventional and RC Monolithic buildings are modelled for every stage and Response spectrum analysis is performed in zone III, with hard soil condition using ETABS version 17 software. The response of the structure is studied in terms of Maximum storey displacement, Maximum storey drift, and Base shear.

It is observed that there is continuous variation in the Maximum storey displacement, Maximum storey drift, and Base shear during each stage of construction and RC monolithic building performs well compared to conventional building when subjected to seismic loads.
\end{abstract}

Keywords: Conventional building, ETABS, RC Monolithic building, Response spectrum analysis, Stage wise construction

\section{INTRODUCTION}

The growing population and urbanization demands tall structures and these tall structures are sensitive to seismic forces. So the buildings are designed to resist earthquake loads. Though the effect cannot be nullified we can reduce the effects on the structure. The recent progress in construction industry has brought RC monolithic building. In this construction method all the structural components are cast in place in one operation by the use of specially designed modular formwork made of aluminium.

Buildings are analysed assuming all the loads are applied in one step but in actual practice structural components like walls, floors finish are provided at different stages of construction to a storey.

\section{OBJECTIVE OF THE WORK}

The primary objective of the work is to analyse the seismic response at every stage of construction for $\mathrm{RC}$ Conventional and Monolithic buildings and to evaluate which structure performs better for seismic loads in seismic zone III with hard soil condition according to Indian standard code IS 1893-2002(part 1)

\section{LITERATURE REVIEW}

Pradeep B, Ambrish G. et.al., (2016) Studied the seismic behaviour of conventional and $\mathrm{RC}$ wall building. The author considered the same plan for $\mathrm{G}+7$ storey conventional and $\mathrm{RC}$ wall building and performed response spectrum analysis using ETABS version 15.2.0 software for both building models in all the 4 zones according to Indian standards code IS:893-2002(part 1). It is concluded that the RC Wall building performs better than conventional building.

Ambrish G. et.al., (2017) Studied seismic response of reinforced concrete monolithic building provided with base isolation. They performed response spectrum analysis to investigate the response of $\mathrm{G}+7$ storey $\mathrm{RC}$ monolithic building provided with base isolation and fixed base in terms of base shear, displacements, storey acceleration and the results are compared. It is observed that zone $\mathrm{V}$ has more response compared to zone II responses for base shear. displacement and storey acceleration. Base isolation is very effective at lessening the seismic response of the structure.

Nagashree V. et.al., (2017) studied the seismic performance on RC Wall structure. The author performed dynamic 
analysis using response spectrum method with 5\% damping, medium soil type and by varying seismic zones .they studied the influence of stiffness irregularities in the form of soft storey, door openings provided at different positions. The performance is evaluated in terms of response spectrum base shear, storey displacement, and modal time period. It is observed that there is very deflection and RC Wall structure enhances the seismic capacity of the structure.

Ahmed Alghuff, Bassam Tayeh, (2019) Performed comparative study of static and response spectrum methods for seismic analysis of regular RC buildings. The author created two structural model using ETABS version 16.1.2 software for regular RC buildings with typical plans. The first model has a total height of $75 \mathrm{~m}$ and the second model has a total height of $24 \mathrm{~m}$. The buildings were analysed using the static and dynamic methods under ASCE7-10 and IBC 2015 provisions and concluded that the displacements obtained using response spectrum method are less compared to equivalent static method.

\section{METHODOLOGY}

In the present investigation, it is assumed that the 24 storeys building with irregular plan is constructed by raising 3 floors at each construction stage and other structural components are added to the underlying floors as the construction process proceeds.

Each stage is modelled for RC conventional and monolithic building and the response spectrum analysis is performed in Zone III with hard soil condition according to IS 18932002(part1) and studied in terms of displacement, drift, and base shear.

\begin{tabular}{|l|l|}
\hline RC Conventional building & \\
\hline Number of floors & 24 \\
\hline Height of each floor & $3 \mathrm{~m}$ \\
\hline Size of beam & $200 \times 500 \mathrm{~mm}$ \\
\hline Wall thickness & $200 \mathrm{~mm}$ \\
\hline Slab thickness & $150 \mathrm{~mm}$ \\
\hline The lift core number & 3 \\
\hline The lift core size & $2.3 \times 3 \mathrm{~m}$ \\
\hline Thickness of lift core & $270 \mathrm{~mm}$ \\
\hline RC Monolithic building & \\
\hline Number of floors & 24 \\
\hline Height of each floor & $3 \mathrm{~m}$ \\
\hline Wall thickness & $200 \mathrm{~mm}$ \\
\hline Slab thickness & $150 \mathrm{~mm}$ \\
\hline The lift core number & 3 \\
\hline
\end{tabular}

\begin{tabular}{|l|l|}
\hline The lift core size & $2.3 \times 3 \mathrm{~m}$ \\
\hline Thickness of lift core & $270 \mathrm{~mm}$ \\
\hline Material properties & \\
\hline Grade of concrete & $\mathrm{M} 30$ \\
\hline Grade of reinforcing steel & $\mathrm{Fe} 500$ \\
\hline Density of concrete block & $17.65 \mathrm{kN} / \mathrm{m}^{3}$ \\
\hline Loading & \\
\hline Floor finish & $1.5 \mathrm{kN} / \mathrm{m}^{2}$ \\
\hline Loads on all rooms & $2 \mathrm{kN} / \mathrm{m}^{2}$ \\
\hline Loads on staircase & $3 \mathrm{kN} / \mathrm{m}^{2}$ \\
\hline Load on corridor, balcony & $3 \mathrm{kN} / \mathrm{m}^{2}$ \\
\hline
\end{tabular}

Table 1: Loads applied on each floor during the process of construction (DL-Dead load, WL-wall load, FF-floor finish, LL- live load)

\begin{tabular}{|c|c|c|c|}
\hline Stages & Storey & Conventional & Monolithic \\
\hline $\begin{array}{c}1 \\
\text { (3 storeys) }\end{array}$ & $\begin{array}{l}1 \\
2 \\
3\end{array}$ & DL & $\mathrm{DL}$ \\
\hline \multirow{2}{*}{$\begin{array}{c}2 \\
\text { (6 storeys) }\end{array}$} & $\begin{array}{l}1 \\
2 \\
3\end{array}$ & $\mathrm{DL}+\mathrm{WL}$ & $\mathrm{DL}$ \\
\hline & $\begin{array}{l}4 \\
5 \\
6\end{array}$ & DL & DL \\
\hline \multirow{3}{*}{$\begin{array}{c}3 \\
\text { (9 storeys) }\end{array}$} & $\begin{array}{l}1 \\
2 \\
3\end{array}$ & $\mathrm{DL}+\mathrm{WL}$ & DL \\
\hline & $\begin{array}{l}4 \\
5 \\
6\end{array}$ & $\mathrm{DL}+\mathrm{WL}$ & DL \\
\hline & $\begin{array}{l}7 \\
8 \\
9\end{array}$ & DL & DL \\
\hline \multirow{4}{*}{$\begin{array}{c}4 \\
(12 \\
\text { storeys) }\end{array}$} & $\begin{array}{l}1 \\
2 \\
3\end{array}$ & $\mathrm{DL}+\mathrm{WL}+\mathrm{FF}$ & $\mathrm{DL}+\mathrm{FF}$ \\
\hline & $\begin{array}{l}4 \\
5 \\
6 \\
\end{array}$ & $\overline{\mathrm{DL}+\mathrm{WL}}$ & DL \\
\hline & $\begin{array}{l}7 \\
8 \\
9\end{array}$ & $\mathrm{DL}+\mathrm{WL}$ & $\mathrm{DL}$ \\
\hline & $\begin{array}{l}10 \\
11 \\
12\end{array}$ & DL & DL \\
\hline 5 & 1 & $\mathrm{DL}+\mathrm{WL}+\mathrm{FF}$ & $\mathrm{DL}+\mathrm{FF}$ \\
\hline
\end{tabular}


International Journal of Engineering Applied Sciences and Technology, 2020

Vol. 5, Issue 6, ISSN No. 2455-2143, Pages 176-182

Published Online October 2020 in IJEAST (http://www.ijeast.com)

\begin{tabular}{|c|c|c|c|}
\hline \multirow[t]{5}{*}{ (15 storeys) } & $\begin{array}{l}2 \\
3\end{array}$ & & \\
\hline & $\begin{array}{l}4 \\
5 \\
6\end{array}$ & $\mathrm{D}+\mathrm{WL}+\mathrm{FF}$ & $\mathrm{DL}+\mathrm{FF}$ \\
\hline & $\begin{array}{l}7 \\
8 \\
9\end{array}$ & $\mathrm{DL}+\mathrm{WL}$ & DL \\
\hline & $\begin{array}{l}10 \\
11 \\
12\end{array}$ & $\mathrm{DL}+\mathrm{WL}$ & DL \\
\hline & $\begin{array}{l}13 \\
14 \\
15\end{array}$ & DL & DL \\
\hline \multirow{6}{*}{$\begin{array}{c}6 \\
\text { (18 storeys) }\end{array}$} & $\begin{array}{l}1 \\
2 \\
3\end{array}$ & $\mathrm{DL}+\mathrm{WL}+\mathrm{FF}$ & $\mathrm{DL}+\mathrm{FF}$ \\
\hline & $\begin{array}{l}4 \\
5 \\
6\end{array}$ & $\mathrm{DL}+\mathrm{WL}+\mathrm{FF}$ & $\mathrm{DL}+\mathrm{FF}$ \\
\hline & $\begin{array}{l}7 \\
8 \\
9\end{array}$ & $\mathrm{DL}+\mathrm{WL}$ & DL \\
\hline & $\begin{array}{l}10 \\
11 \\
12\end{array}$ & $\mathrm{DL}+\mathrm{WL}$ & $\overline{\mathrm{DL}}$ \\
\hline & $\begin{array}{l}13 \\
14 \\
15 \\
\end{array}$ & $\mathrm{DL}+\mathrm{WL}$ & DL \\
\hline & $\begin{array}{l}16 \\
17 \\
18\end{array}$ & $\mathrm{DL}$ & DL \\
\hline \multirow[t]{7}{*}{$\begin{array}{c}7 \\
\text { (21 storeys) }\end{array}$} & $\begin{array}{l}1 \\
2 \\
3 \\
\end{array}$ & $\mathrm{DL}+\mathrm{WL}+\mathrm{FF}$ & $\mathrm{DL}+\mathrm{FF}$ \\
\hline & $\begin{array}{l}4 \\
5 \\
6 \\
\end{array}$ & $\mathrm{DL}+\mathrm{WL}+\mathrm{FF}$ & $\mathrm{DL}+\mathrm{FF}$ \\
\hline & $\begin{array}{l}7 \\
8 \\
9\end{array}$ & $\mathrm{DL}+\mathrm{WL}+\mathrm{FF}$ & DL+FF \\
\hline & $\begin{array}{l}10 \\
11 \\
12\end{array}$ & $\mathrm{DL}+\mathrm{WL}+\mathrm{FF}$ & $\mathrm{DL}+\mathrm{FF}$ \\
\hline & $\begin{array}{l}13 \\
14 \\
15 \\
\end{array}$ & $\mathrm{DL}+\mathrm{WL}$ & DL \\
\hline & $\begin{array}{l}16 \\
17 \\
18 \\
\end{array}$ & $\mathrm{DL}+\mathrm{WL}$ & DL \\
\hline & $\begin{array}{l}19 \\
20 \\
21\end{array}$ & $\mathrm{DL}$ & $\mathrm{DL}$ \\
\hline 8 & 1 & $\mathrm{DL}+\mathrm{WL}+\mathrm{FF}$ & $\mathrm{DL}+\mathrm{FF}$ \\
\hline
\end{tabular}

\begin{tabular}{|c|c|c|c|}
\hline \multirow[t]{8}{*}{ (24 storeys) } & $\begin{array}{l}2 \\
3\end{array}$ & & \\
\hline & $\begin{array}{l}4 \\
5 \\
6\end{array}$ & $\mathrm{DL}+\mathrm{WL}+\mathrm{FF}$ & $\mathrm{DL}+\mathrm{FF}$ \\
\hline & $\begin{array}{l}7 \\
8 \\
9\end{array}$ & $\mathrm{DL}+\mathrm{WL}+\mathrm{FF}$ & $\mathrm{DL}+\mathrm{FF}$ \\
\hline & $\begin{array}{l}10 \\
11 \\
12\end{array}$ & $\mathrm{DL}+\mathrm{WL}+\mathrm{FF}$ & $\mathrm{DL}+\mathrm{FF}$ \\
\hline & $\begin{array}{l}13 \\
14 \\
15\end{array}$ & $\mathrm{DL}+\mathrm{WL}+\mathrm{FF}$ & $\mathrm{DL}+\mathrm{FF}$ \\
\hline & $\begin{array}{l}16 \\
17 \\
18\end{array}$ & $\mathrm{DL}+\mathrm{WL}$ & $\overline{\mathrm{DL}}$ \\
\hline & $\begin{array}{l}19 \\
20 \\
21\end{array}$ & $\mathrm{DL}+\mathrm{WL}$ & DL \\
\hline & $\begin{array}{l}22 \\
23 \\
24 \\
\end{array}$ & $\mathrm{DL}+\mathrm{WL}$ & DL \\
\hline \multirow{8}{*}{$\begin{array}{c}9 \\
\text { (24 storeys, } \\
\text { Ready to } \\
\text { occupy) }\end{array}$} & $\begin{array}{l}1 \\
2 \\
3\end{array}$ & $\mathrm{DL}+\mathrm{WL}+\mathrm{FF}$ & $\mathrm{DL}+\mathrm{FF}$ \\
\hline & $\begin{array}{l}4 \\
5 \\
6 \\
\end{array}$ & $\mathrm{DL}+\mathrm{WL}+\mathrm{FF}$ & $\mathrm{DL}+\mathrm{FF}$ \\
\hline & $\begin{array}{l}7 \\
8 \\
9\end{array}$ & $\mathrm{DL}+\mathrm{WL}+\mathrm{FF}$ & DL+FF \\
\hline & $\begin{array}{l}10 \\
11 \\
12 \\
\end{array}$ & $\mathrm{DL}+\mathrm{WL}+\mathrm{FF}$ & $\mathrm{DL}+\mathrm{FF}$ \\
\hline & $\begin{array}{l}13 \\
14 \\
15 \\
\end{array}$ & $\mathrm{DL}+\mathrm{WL}+\mathrm{FF}$ & $\mathrm{DL}+\mathrm{FF}$ \\
\hline & $\begin{array}{l}16 \\
17 \\
18\end{array}$ & DL+WL+FF & DL+FF \\
\hline & $\begin{array}{l}19 \\
20 \\
21\end{array}$ & $\mathrm{DL}+\mathrm{WL}+\mathrm{FF}$ & $\mathrm{DL}+\mathrm{FF}$ \\
\hline & $\begin{array}{l}22 \\
23 \\
24 \\
\end{array}$ & $\mathrm{DL}+\mathrm{WL}+\mathrm{FF}$ & $\mathrm{DL}+\mathrm{FF}$ \\
\hline \multirow{3}{*}{$\begin{array}{c}10 \\
\text { (24storeys, } \\
\text { Occupied) }\end{array}$} & $\begin{array}{l}1 \\
2 \\
3\end{array}$ & $\begin{array}{l}\mathrm{DL}+\mathrm{WL}+\mathrm{FF}+ \\
\mathrm{LL}\end{array}$ & $\mathrm{DL}+\mathrm{FF}+\mathrm{LL}$ \\
\hline & $\begin{array}{l}4 \\
5 \\
6 \\
\end{array}$ & $\begin{array}{l}\mathrm{DL}+\mathrm{WL}+\mathrm{FF}+ \\
\mathrm{LL}\end{array}$ & $\mathrm{DL}+\mathrm{FF}+\mathrm{LL}$ \\
\hline & 7 & $\mathrm{DL}+\mathrm{WL}+\mathrm{FF}+$ & $\mathrm{DL}+\mathrm{FF}+\mathrm{LL}$ \\
\hline
\end{tabular}




\begin{tabular}{|l|l|l|l|}
\hline & 8 & LL & \\
9 & & \\
\cline { 2 - 4 } & 10 & DL+WL+FF+ & DL+FF+LL \\
11 & LL & \\
12 & & \\
\cline { 2 - 4 } & 13 & DL+WL+FF+ & DL+FF+LL \\
14 & LL & \\
15 & & DL+FF+LL \\
\cline { 2 - 4 } & 16 & DL+WL+FF+ & \\
17 & LL & \\
18 & & DL+FF+LL \\
\hline 19 & DL+WL+FF+ & \\
20 & LL & \\
21 & & \\
\cline { 2 - 4 } & 22 & DL+WL+FF+ & DL+FF+LL \\
23 & LL & \\
24 & & \\
\hline
\end{tabular}

V. ETABS MODELLING

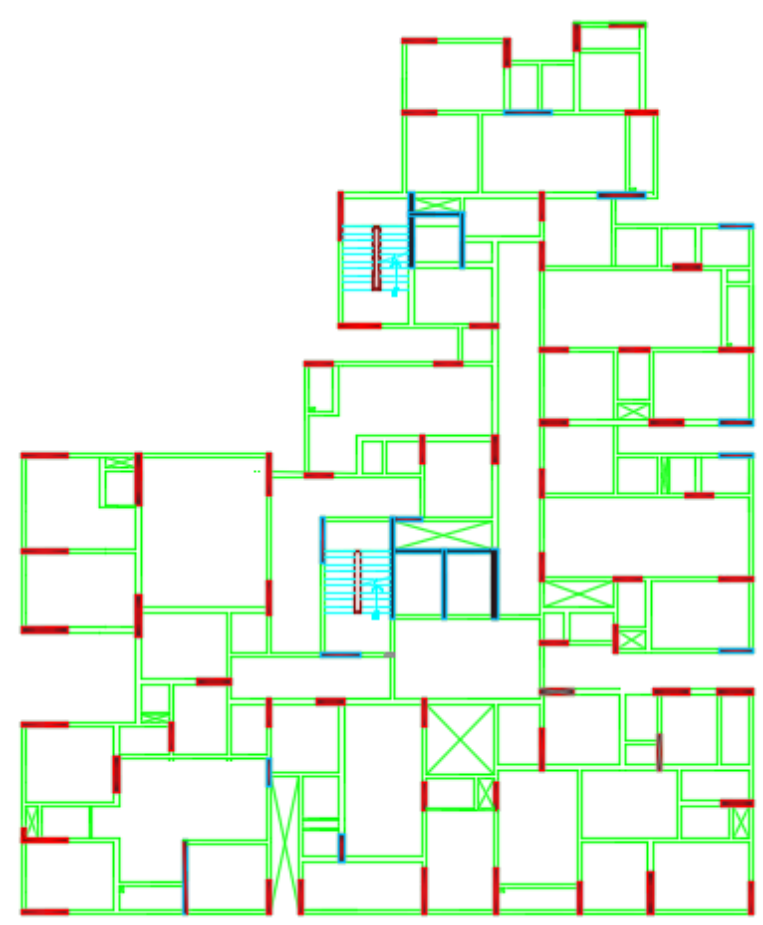

Fig 1: Plan of building

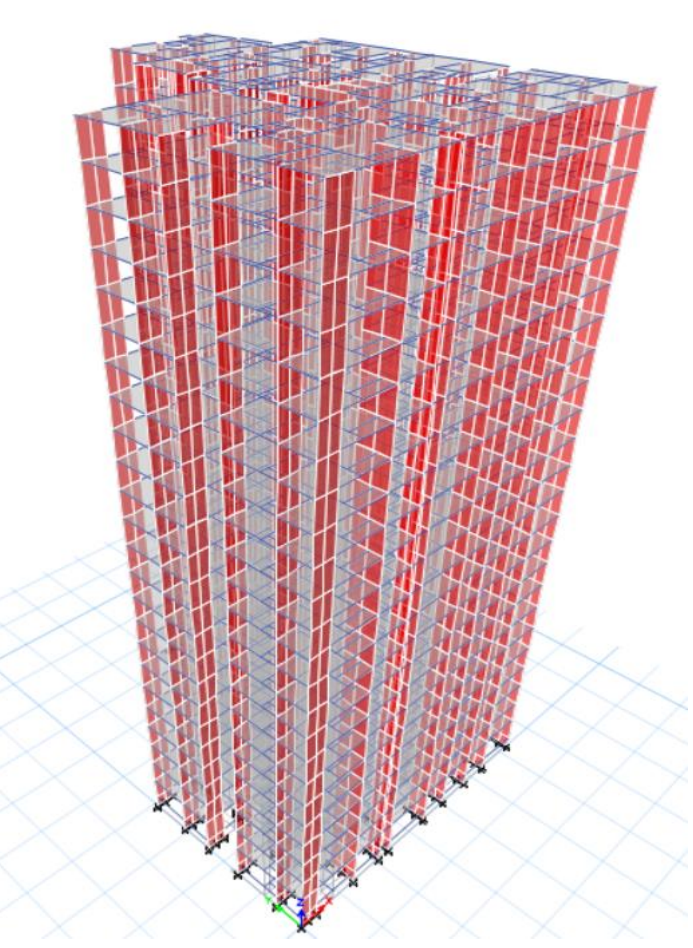

Fig 2: 3D model of RC Conventional building

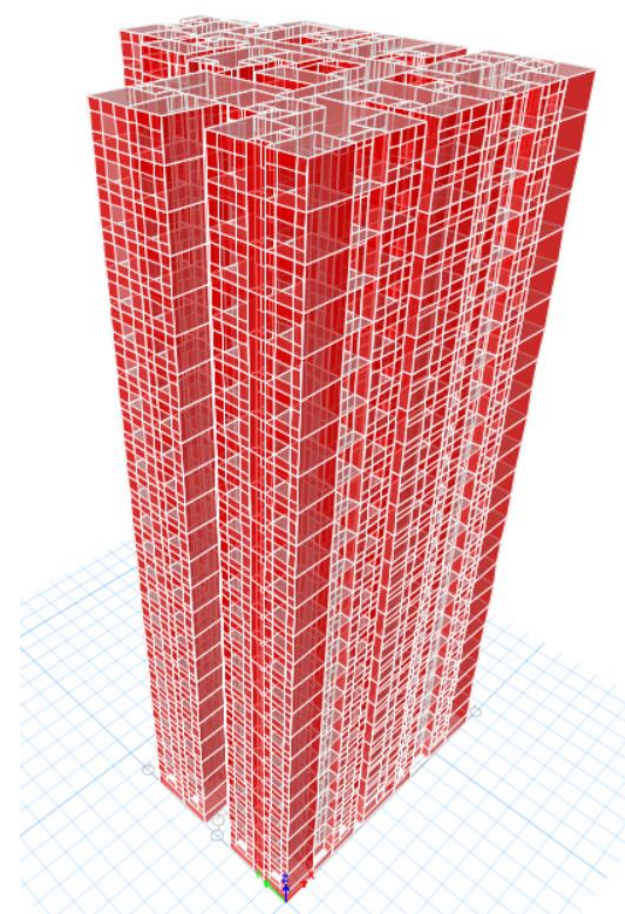

Fig 3: 3D modelling of RC Monolithic building 


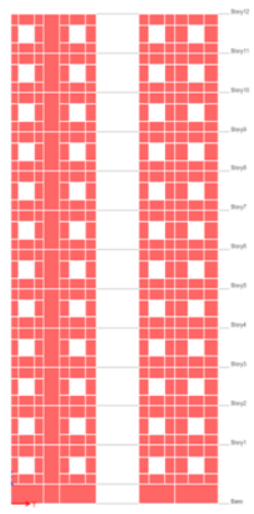

Stage 4 (12 storeys)

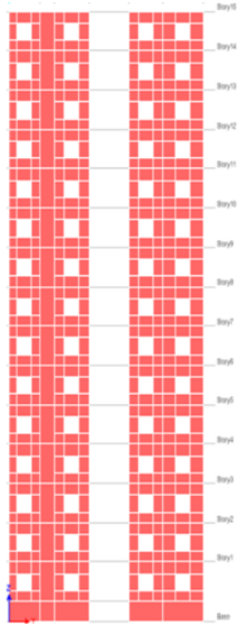

stage 5 (15 storeys)

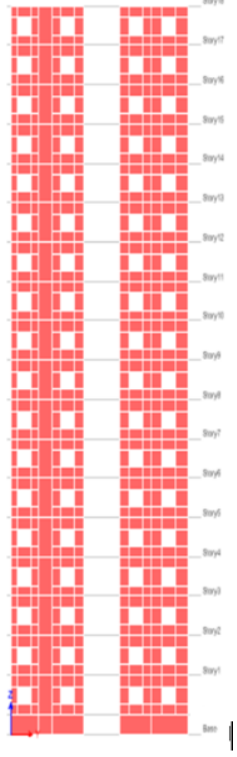

stage 6(18 storeys)

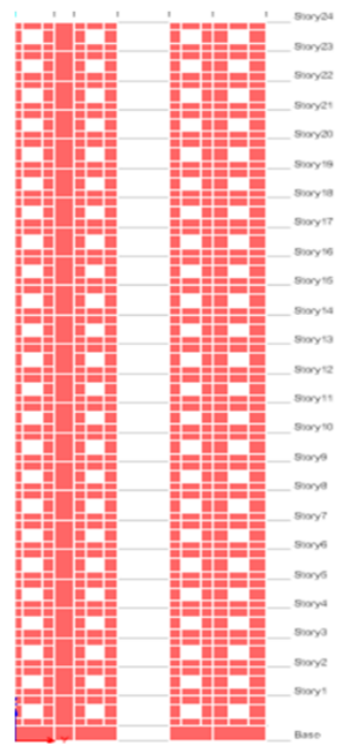

Stage 8 (24 storeys)|

\section{RESULTS}

The RC conventional and monolithic building is analysed using response spectrum method at every construction stage according to IS 1893-2002(part 1) and the responses are studied in terms of storey displacement, storey drift, Base shear.
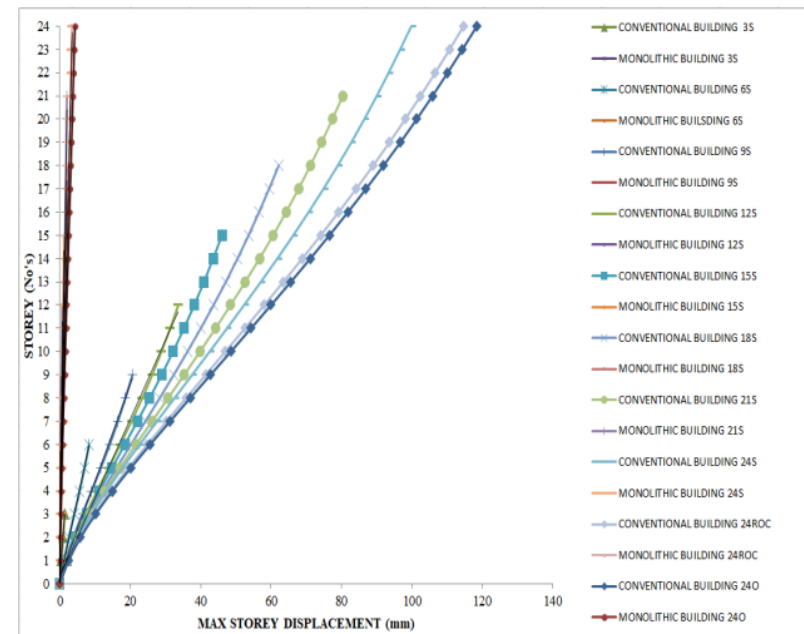

Chart 1: Variation in storey displacement at every stage of construction - RS X

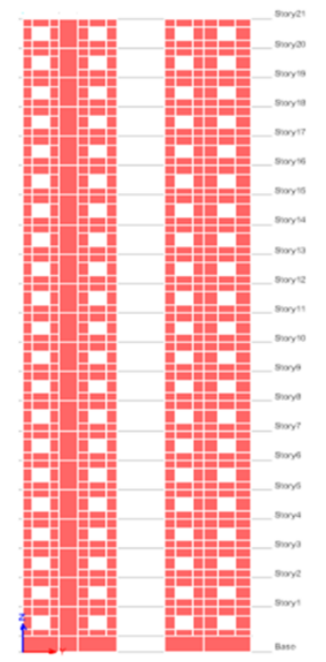

Stage 7 (21 storeys)
Fig 2: Stages of construction

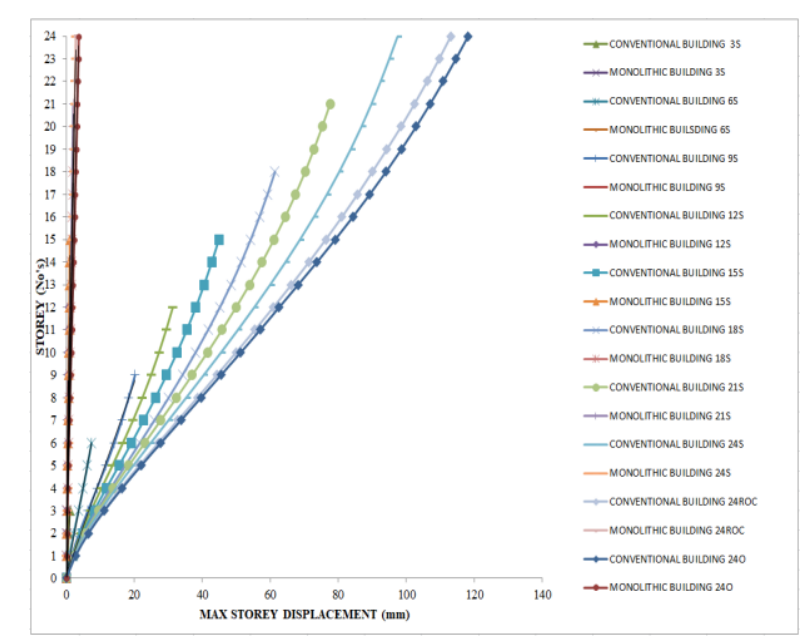

Chart 2: Variation in storey displacement at every stage of construction - RS Y 


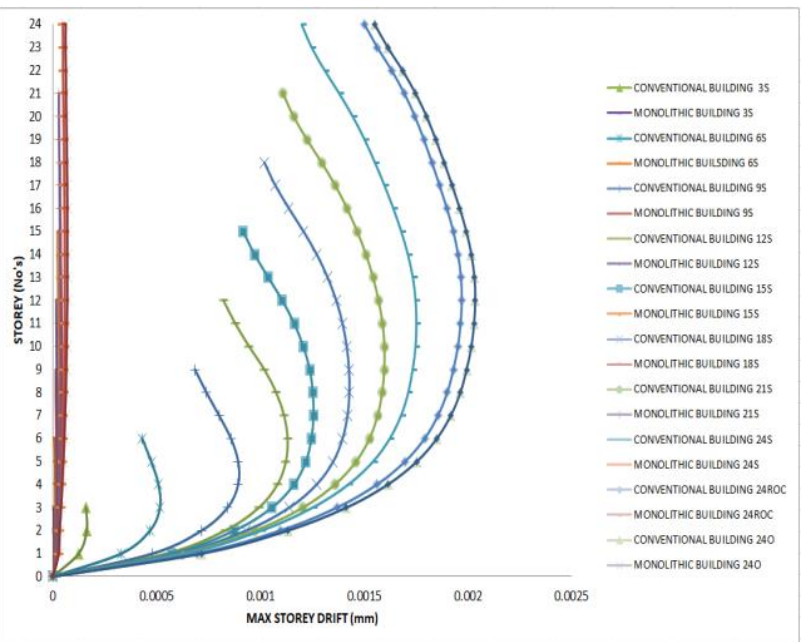

Chart 3: Variation in storey drift at every stage of construction - RS X

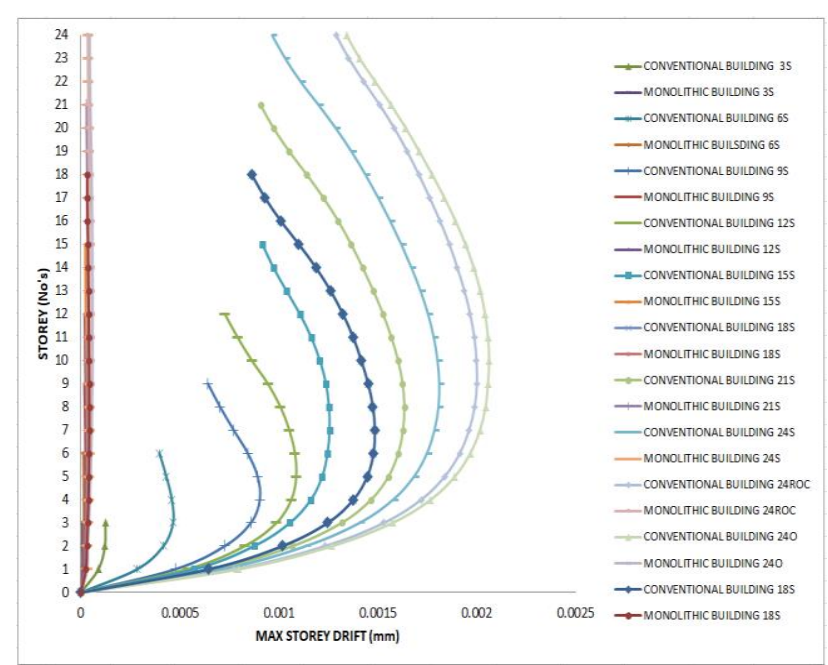

Chart 4: Variation in storey drift at every stage of construction - RS Y

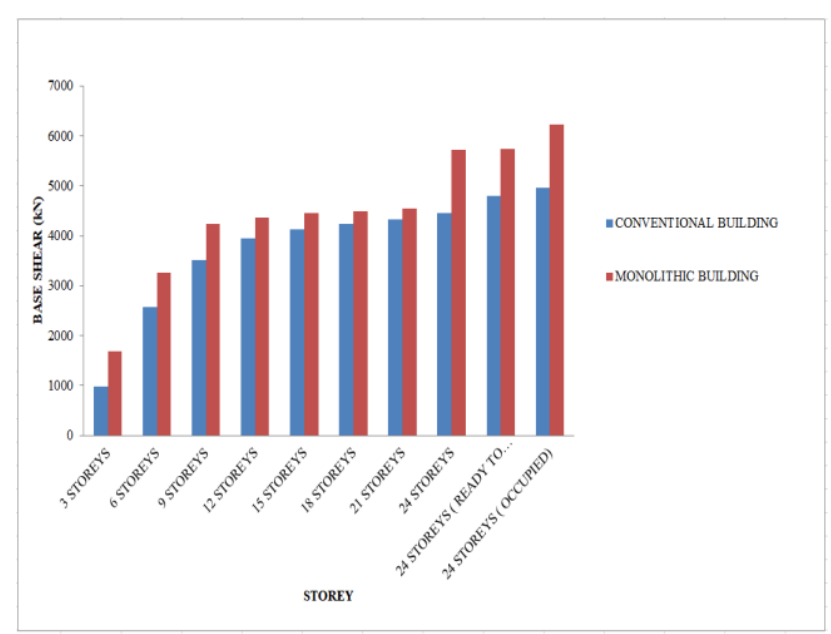

Chart 5: Variation in Base shear at every stage of construction - RS X

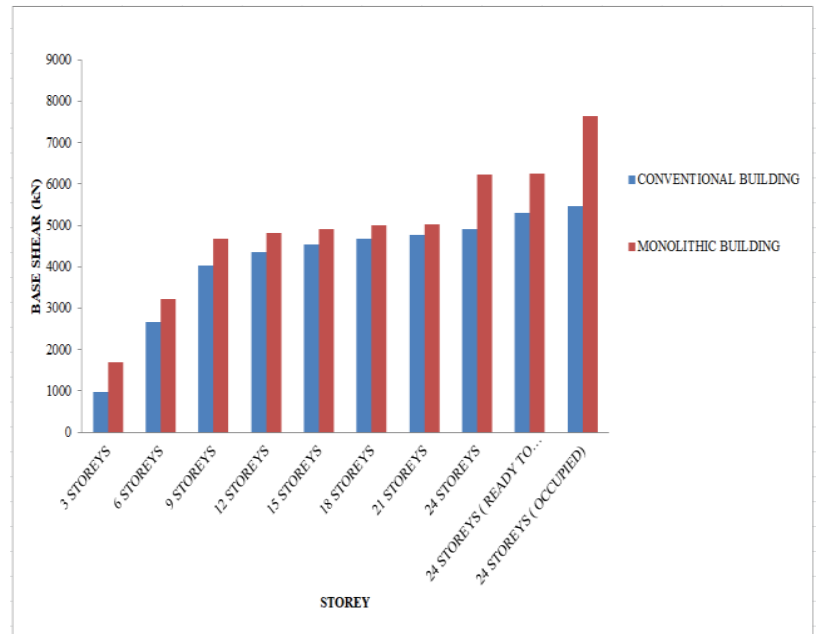

Chart 6: Variation in Base shear at every stage of construction - RS Y

\section{CONCLUSIONS}

An effort is made to examine the structural response of 24 storeys RC Conventional and Monolithic building at each stage of construction for seismic loads. From the analysis results it can be inferred that,

1. In RC Conventional buildings, maximum storey displacement and maximum storey drift is $97 \%$ greater than Monolithic buildings. As a result, RC Monolithic building is more robust to seismic loads

2. Base shear is greater in RC monolithic building than conventional building.

3. Storey displacement is induced by the lateral force on each floor level of the structure. Lateral displacement on the top floor would be more significant. The disparity in maximum storey displacement and Maximum storey drift during the construction of $24^{\text {th }}$ floor and the fully occupied 24 storey building (all the loads are assumed to be acting in one step) is $15 \%$ for conventional $\mathrm{RC}$ building and $18 \%$ for monolithic building.

4. The difference in base shear during the construction of $24^{\text {th }}$ floor and the entirely occupied 24 -storey building(all loads are presumed to work in one stage) is $10 \%$ more for conventional building in both the directions and $8 \%$ more in $\mathrm{x}$ direction, $18.5 \%$ more in $\mathrm{y}$ direction for $\mathrm{RC}$ monolithic building.

5. It is found from the above graph and discussion that there is significant variation in storey responses on the construction of each floor and other structural components. 


\section{International Journal of Engineering Applied Sciences and Technology, 2020 \\ Vol. 5, Issue 6, ISSN No. 2455-2143, Pages 176-182 \\ Published Online October 2020 in IJEAST (http://www.ijeast.com)}

\section{REFERENCES}

[1]. B Pradeep, KS Shiva Kumar, G Ambrish, (2016). "Study of seismic behaviour of conventional and RC wall building", IJRET, Vol. 5, Issue 6, Pg.467-474, eISSN: 2319-1163|pISSN:2321-7308.

[2]. G Ambrish, H Sharada Bai, (2017). "Seismic response of reinforced concrete monolithic building provided with base isolation", IJARSE, Vol. 6, Issue 12, Pg.1543-1558, ISSN: 2319-8354.

[3]. Nagashree V, D S Sandeep Kumar, (2017). "Seismic Performance Study ON RC Wall Structures”, IRJET, Vol. 4, Issue 6, Pg.2833-2836, ISSN: 2395-0056.

[4]. Ahmed Y. Alghuff, Bassam Tayeh, (2019). "Comparative study of static and response spectrum methods for seismic analysis of regular $\mathrm{RC}$ buildings",J.Applied Sci., 19(5):495-503, DOI: $10.3923 /$ jas. 2019, ISSN: 1812-5654

[5]. Nikitha Patel, Namrata Verma, (2017). "A study in monolithic structure", IJMPE, pp. 84-91, Vol. 05, Issue 11, ISSN: 2320-2092

[6]. Amal R. Vora, Prof.P.G Patel, (2015). "A comparative study of conventional structure system with monolithic structural system", IJSTE, Vol. 01,Issue 11, Pg.157-160, ISSN (online):2349-784X

[7]. H B Santosh, D Prakash, Naveen Kumar, (2016). "Seismic analysis and comparison between monolithic RC structure bare and masonry structure", IJRET, Vol.05, Issue07, Pg.375-378, ISSN:2319-1163

[8]. Haaris M. Mal, Prof. Umang Parekh, (2016). "Comparative study of Conventional structure with Monolithic structure", IJSR,Vol. 05, Issue 05, Pg 866-868, ISSN(online):2319-7064

[9]. Devang Gohel, Dr. JayeshKumar Pitroda, (2017). "A critical literature review on comparative study of conventional structure with monolithic structure", IJCRT, Vol. 05, Issue 04,Pg 2220-2223, ISSN:23202882

[10]. Girum Mindaye, Dr. Shaik Yajdani, (2016). "Seismic analysis of a multi-storey RC Frame building in different seismic zones", IJIRSET, Vol. 05, Issue 09, DOI:10.15680/IJIRSET.201820706019, ISSN(online):2319-8753

[11]. Devang Gohel , Dr. JayeshKumar Pitroda , Amithkumar D. Raval (2018). " Comparative study of Monolithic structure over Conventional structure, Monolithic structure: A Case study at Vadodara city" IJTIMES, Vol.4, Issue 5,Pg 420-424, ISSN: 2455-2585

[12]. H. Gonzales, F. Lopez-Almansa (2011) "Seismic performance of buildings with thin RC bearing walls"ELSEVIER,DOI:10.1016/j.engstruct.2011.10.007

[13]. IS-1893-2002(part 1) criteria for Earthquake Resistant design of structure.

[14]. IS-875(part 2)-1987 used for design loads (other than earthquake) for building and structure.
[15]. IS-875(part 2)-1987 used for design loads (other than earthquake) for building and structure.

[16]. IS-456:2000 used for plain and reinforced concrete. 